\title{
ZUR GARTENKÜNSTLERISCHEN LEHRE AN DER GARTENBAUSCHULE DES Gartenbauverbandes FÜr das Königreich SaCHSEN
}

\author{
Ingolf Hohlfeld
}

\section{8-1895}

$\mathrm{D}$ ie erste Initiative zur Gründung einer »Schule für die theoretische Ausbildung tüchtiger junger Gärtnergehilfen " ging von der Hauptversammlung des »Verbandes sächsischer Gartenbauvereine" am 6. Februar 1888 aus. Es wurde eine Kommission aus sieben namhaften Berufskollegen gebildet, die bei der Verbandsversammlung 1889 folgende Empfehlungen $\mathrm{zu}$ einer Dreiteilung der gärtnerischen Aus- und Weiterbildung aussprach:

- Vermehrung der an einzelnen Orten bereits bestehenden gärtnerischen Fachschulen für Lehrlinge;

- Errichtung einer Gartenbauschule für Gehilfen mit besserer Schulbildung;

- Gründung einer Akademie für Gartenkunst.

Max Bertram (1849-1914), der erste Direktor der Gartenbauschule, schrieb dazu im ersten Bericht über die Gartenbauschule des Gartenbau-Verbandes für das Königreich Sachsen:

»Man hielt es für durchaus nothwendig, entsprechend dem klaren Unterschied zwischen dem Gartenbau, dem die Anzucht und Kultur des Pflanzenmaterials obliegt, und der Gartenkunst, welche sich mit der künstlerischen Verwendung der Pflanzen im Park und Garten befaßt, zwei verschiedene Institute zu begründen und je nach dem Zwecke, den einen oder den anderen Zweig des gemeinsamen Stammes, der Gärtnerei überhaupt, beim Unterricht in den Vordergrund zu stellen ${ }^{1}{ }^{1}$

Die Verbandsversammlung stimmte diesen Empfehlungen zu. In der Folge wurde dem Königlichen Ministerium des Innern eine
Denkschrift übergeben, die auf die Errichtung einer Gartenbauschule und einer Akademie für Gartenkunst in Dresden abzielte. Das Ministerium sagte hierauf die Unterstützung für eine vom Verband zu begründende Gartenbauschule zu, setzte aber gleichzeitig die Entscheidung zur Errichtung einer Hochschule für Gartenkunst aus.

Bis 1892 waren die Vorbereitungen (Entwürfe für Lehrpläne, für die Schulordnung, das Schulregulativ u. ä.) soweit gediehen, dass das Ministerium am 12. April 1892 die Eröffnung der Schule genehmigte. Bereits am 15. Mai 1892 wurde die Schule in der Hassestraße 1 in Striesen eröffnet. Die Eröffnung erfolgte mit sieben Schülern, davon drei aus Sachsen. Folgende Lehrer bestritten den Unterricht:

- Direktor Max Bertram aus Blasewitz, für Feldmessen, Freihand- und Planzeichnen;

- Herr Otto Lämmerhirt aus Dresden, Garten-Inspector und Geschäftsführer des Landesobstbauvereins, für Obst- und Weinbau;

- Herr Otto Meckwitz aus Bautzen, für Garten- und Gemüsebau;

- Herr Dr. phil. Arno Naumann aus Dresden, Assistent bei der Königlichen Technischen Hochschule und diplomierter Chemiker, für Botanik, Chemie, Physik und Wetterkunde;

- Herr Ernst Moritz Trenkler, Lehrer an der II. Bürgerschule und staatlich geprüfter Lehrer für englische Sprache, für den englischen Unterricht;

- Herr Hermann Steinert, Lehrer an der VIII. Bürgerschule und staatlich geprüfter 
Lehrer für französische Sprache, für den Unterricht im Französischen;

- Herr Georg Jüde, Lehrer an der Evangelischen Freischule und Dirigent der Gartenbauschule der Genossenschaft »Flora", für Rechnen und Buchführung;

- Herr Ernst Robert Mißbach, Lehrer an der IX. Bezirksschule, für Geometrie und Zoologie;

- Herr Hermann Stöcklein, Ingenieur aus Striesen bei Dresden, vom II. Semester ab für den Unterricht in Gewächshausbau.

Die Schule befand sich in gemieteten Räumen eines Wohnhauses. Ein gärtnerisches Umfeld wie Gewächshäuser, Pflanzensammlungen oder ähnliches fehlte. Die Schule sollte den Ansatz verfolgen, nur theoretischen Unterricht, ergänzt mit Exkursionen, zu bieten. Praktische Arbeiten sollten keine Rolle spielen, da diese die Schüler schon in der Lehre kennengelernt hatten. Diese Reduzierung auf die Theorie, die sich letztlich durchaus bewährte, traf bei vielen Gärtnern zunächst auf Unverständnis und war anfangs Quelle für eine verbreitete Skepsis gegenüber der Schule.

Die Ausbildung war als zweijähriger Unterricht konzipiert. Der Lehrplan zeigt, dass der Zierpflanzenbau, der Gemüse-, Obstund Weinbau, Handelsfragen und Sprachen, Botanik, Physik und Chemie sowie der Gewächshausbau Schwerpunkte der Ausbildung waren.

Zur Gartenkunst äußerte sich Bertram im ersten Bericht über die Gartenbauschule des Gartenbau-Verbandes für das Königreich Sachsen folgendermaßen:

»Eine gewisse Übung im Plan- und Freihandzeichnen, ingleichen Fertigkeiten im einfachen Feldmessen und Nivelliren ist jedem jungen Gärtner von Nutzen und besonders denen unentbehrlich, welche sich der Ausübung der Landschaftsgärtnerei widmen . $^{2}$

Der Unterricht zur Landschaftsgärtnerei umfasste in der unteren Stufe fünf Wochenstunden (Zeichnen und Feldmessen) und in der oberen Stufe sieben Wochenstunden
(Zeichnen und Feldmessen, Gartenanlage). Zählt man Geometrie und Botanik hinzu, waren es zehn respektive dreizehn Wochenstunden.

Die schwerpunktmäßige Hinwendung auf den Produktionsgartenbau wurde auch in einem Informationsblatt der Gartenbauschule aus den Anfangsjahren betont:

"Sitz der Gartenbauschule ist Dresden, ihr Zweck: jungen Gärtnern durch planmäßige Unterweisung eine theoretische Ausbildung zu vermitteln, die ihnen für die spätere erfolgreiche Ausübung ihres Berufes förderlich ist. Wie will sie diesen Zweck erreichen?

Sie berücksichtigt bei dem Aufbau ihres Lehrplanes alle Verhältnisse, die dem jungen Manne bei Gründung, Einrichtung und Erbauung einer Gärtnerei, bei Ausführung der verschiedensten Culturen und bei dem Vertriebe der fertigen Erzeugnisse begegnen können «. $^{3}$

Zur Landschaftsgärtnerei hieß es nach der Darstellung des Unterrichtes für den Produktionsgartenbau:

»[Wer] sich durch Befähigung und Neigung darauf hingewiesen fühlt, die Landschaftsgärtnerei zum Felde seiner Thätigkeit zu wählen, vermag sich durch die bisher genannten Unterrichtsfächer die erforderliche Allgemeinbildung und Pflanzenkenntnis zu erwerben. Im Besonderen aber sucht die Anstalt ihre Zöglinge durch Unterweisung in Geometrie, Feldmessen, Freihand-, Plan-, Linear- und Landschaftszeichnen $z u$ nützlichen Hilfskräften für die Gartenkünstler heranzubilden und ihnen zu ermöglichen, innerhalb gewisser Grenzen später auch auf diesem Gebiete Selbständiges zu leisten «. ${ }^{4}$ Es ergibt sich als Fazit, dass zu Beginn der Ausbildung an der neuen Gartenbauschule die Landschaftsgärtnerei zwar behandelt wurde, aber keineswegs eine besondere Vertiefung erfuhr. Gegenüber dem ursprünglichen Ziel, eine Hochschule für Gartenkunst ins Leben zu rufen, waren erhebliche Abstriche zu verzeichnen, was zum Teil auch damit zusammenhängen dürfte, dass man im 


\section{Stundenplan für das Sommer:Semeiter 1901.}

리 $\mathfrak{a} \mathfrak{x}$ m $\mathfrak{i} \mathbf{t} \mathfrak{t} \mathfrak{a} \mathfrak{g}$.

\begin{tabular}{|c|c|c|c|c|c|c|c|c|c|c|c|}
\hline \multicolumn{2}{|c|}{ Nontag } & \multicolumn{2}{|c|}{ Dicustag } & \multicolumn{2}{|c|}{ Mittmod) } & \multicolumn{2}{|c|}{ Donnerstag } & \multicolumn{2}{|c|}{ Freitag } & \multicolumn{2}{|c|}{ Sonnabcti } \\
\hline I & II & I & II & I & II & I & II & I & II & I & II \\
\hline \multirow[t]{2}{*}{ 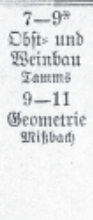 } & \multirow[t]{2}{*}{$\begin{array}{c}9-11^{*} \\
\text { Semiijebau } \\
\text { 2ammas }\end{array}$} & \multirow[t]{2}{*}{ 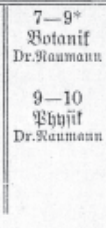 } & \multirow[b]{2}{*}{$\begin{array}{c}\text { 10-12* } \\
\text { Botanif } \\
\text { Dr.9aumaum }\end{array}$} & \multirow{2}{*}{ 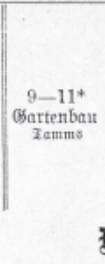 } & \multirow{2}{*}{ 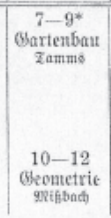 } & \multirow{2}{*}{ 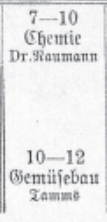 } & \multirow{2}{*}{ 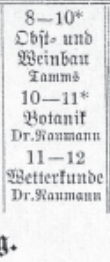 } & \multirow{2}{*}{\multicolumn{2}{|c|}{ 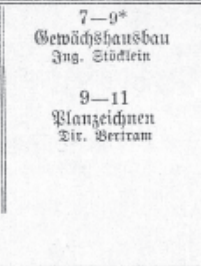 }} & \multirow[t]{2}{*}{$\begin{array}{c}7-8 \\
\text { 8ิtomefien }\end{array}$} & \multirow[t]{2}{*}{$\begin{array}{c}9-11 \\
\text { gelomeien }\end{array}$} \\
\hline & & & & & & & & & & & \\
\hline 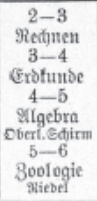 & $\begin{array}{c}4-5 \\
\text { Dentifid } \\
\text { gitebel }\end{array}$ & $\begin{array}{c}3-4^{1 /} / 2 \\
\text { Jrantjifitid) } \\
\text { \&offanann }\end{array}$ & 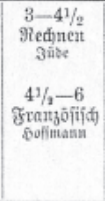 & 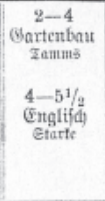 & $\begin{array}{c}21 / 2-4 \\
\text { Engfifid) } \\
\text { Etarte } \\
4-6^{*} \\
\text { Sartentbaut } \\
\text { इatumat }\end{array}$ & 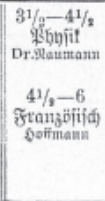 & 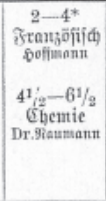 & \begin{tabular}{|c|}
$-41 / 2$ \\
Earten= \\
Qntagen \\
Dir, Sertram \\
$4-51 / 2$ \\
Englifid) \\
Etarte \\
\end{tabular} & $\begin{array}{l}21 / 9-4 \\
\text { Englifid) } \\
\text { Etarte } \\
4-5 \\
4-5 \\
\text { Redfuen } \\
\text { 3ube }\end{array}$ & 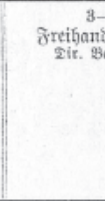 & $\begin{array}{l}-5 \\
\text { gieidguent } \\
\text { ctraik. }\end{array}$ \\
\hline
\end{tabular}

\section{Stumdenplan fïr das Winter:Semeiter 1901-1902.}

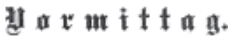

\begin{tabular}{|c|c|c|c|c|c|c|c|c|c|c|c|}
\hline \multicolumn{2}{|c|}{ Miontag } & \multicolumn{2}{|c|}{ Dienstag } & \multicolumn{2}{|c|}{ Mittwody } & \multicolumn{2}{|c|}{ Donuerstag } & \multicolumn{2}{|c|}{ ซ̃reitag } & \multicolumn{2}{|c|}{ Sontubent } \\
\hline I & II & I & II & I & II & I & II & I & II & I & II \\
\hline $\begin{array}{c}10-12 \\
\text { Cbits utb } \\
\text { 2Eeinbaut } \\
\text { rammo }\end{array}$ & 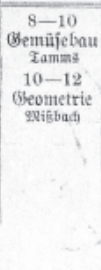 & 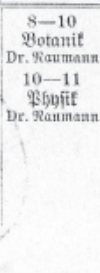 & $\begin{array}{c}12-1 \\
\text { Botenit } \\
\text { Dr. Yaumann }\end{array}$ & 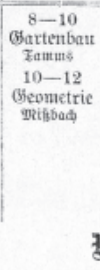 & $\begin{array}{c}10-12 \\
\text { Sortentbaut } \\
\text { Zamums }\end{array}$ & 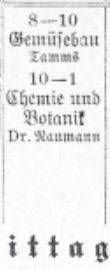 & 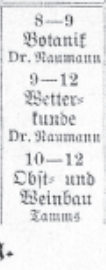 & 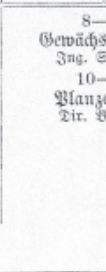 & 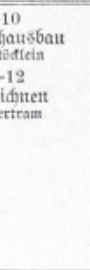 & $\begin{array}{c}9- \\
\text { ơreihant: } \\
\text { Tii., }\end{array}$ & idguent \\
\hline 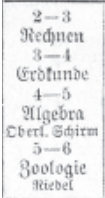 & $\begin{array}{c}4-5 \\
\text { Deutidi } \\
\text { Stieorl }\end{array}$ & 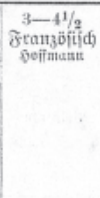 & 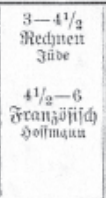 & 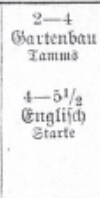 & $\begin{array}{c}21 / 2-4 \\
\text { Englijif } \\
\text { Etarte } \\
4-6 \\
\text { Giactenbau } \\
\text { Zammab }\end{array}$ & 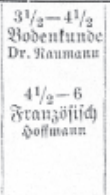 & 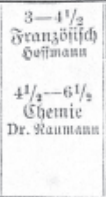 & $\begin{array}{c}2-4 \\
\text { Garten= } \\
\text { antfagen } \\
\text { Dir, } 20 r \text { rtram } \\
4-51 / 2 \\
\text { Engliid) } \\
\text { Etartc }\end{array}$ & 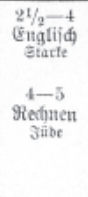 & 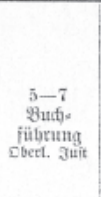 & 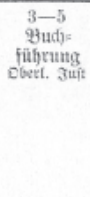 \\
\hline
\end{tabular}

1 | Stundenpläne für das Sommersemester 1901 bzw. Wintersemester 1901/02. (9. Bericht über die Gartenbauschule des Gartenbau-Verbandes für das Königreich Sachsen).

Berufsstand zunächst immer noch auf die Gründung einer Hochschule für Gartenkunst als zweiten Schritt hoffte.

Die Lehrmittelausstattung der Schule war anfangs schlecht, was auch den Unterricht in der Landschaftsgärtnerei berührte. So wurde in einem Schreiben an das Ministerium vom 8. Februar 1893 festgestellt, dass die Messgeräte für das Feldmessen bisher ausgeliehen werden, weshalb 425,40 Mark für Anschaffung vorgesehen wurden. Auch für den Unterricht im Planzeichnen fehlte das Nötigste:

»Um dem gänzlichen Mangel für das Planzeichnen zu begegnen hat sich der Direktor der Anstalt an den Verleger seines Werkes >Das gärtnerische Planzeichnen gewandt. Demnach sollen der Schule die acht hauptsächlichsten Übungsblätter des Werkes in 20 Serien á 2,50 Mark geliefert werden. [...] Überdies sollen von 
dem gesamten Werke [...] 3 Exemplare angeschafft werden, welche unter Berücksichtigung von $5 \%$ Buchhändlerrabatt 34,20 Mark kosten «. ${ }^{5}$

Außerdem wurde auf die schlechte Haushaltslage der Schule hingewiesen. An dieser hat sich während ihres gesamten Bestehens trotz verschiedenster Bemühungen um Verbesserung nichts geändert. Jährlich wurden Zuschüsse des Ministeriums in Höhe von durchschnittlich 5.000 Mark benötigt.

\section{6-1898}

Nach vierjährigem Bestehen der Schule strebte das Ministerium des Innern eine Bewertung ihrer Tätigkeit an. Es erging eine Einladung an einige Vertreter des Gartenbaus zu einem Gespräch am 4. November 1896. Der Vorsitzende, der Geheime Rat Vodel, formulierte zu Beginn des Gespräches das Ziel, »aus dem Munde hervorragender Fachkenner ein Urtheil zur Wirksamkeit und Leistungsfähigkeit der Schule $\mathrm{zu}$ hören $[\ldots]$ «. $^{6}$ Seitens des Berufstandes nahmen an dem Gespräch teil:

- Friedrich Bouché, Dresden, Obergartendirektor,

- Privatus Conrad Schulze, Blasewitz,

- Rudolph Seidel, Laubegast, Kunst- und Handelsgärtner,

- Herr Eck, Blasewitz, Gartenbauingenieur,

- Herr Werner, Stadt-Garteninspektor in Chemnitz,

- Herr Dr. Blochwitz, Stadtrat in Dresden,

- Hermann Schmidt, Leipzig-Möckern, Kunst- und Handelsgärtner.

Aus diesem Kreis wurden recht unterschiedliche Auffassungen zur Arbeit der Schule vertreten. Während Bouché, Schmidt und Seidel ein grundsätzlich positives Urteil abgaben, erläuterte Werner, der Chemnitzer Stadtrat glaube, die Schule verfehle ihr Ziel, indem sie eine Art Akademie darstellen wolle - weil in der Schulordnung alle Veranstaltungen, die das studentische Verbindungswesen nachahmen, untersagt waren - und auch der hohe Stundenanteil für Geometrie, Feldmessen und Freihandzeichnen passe nicht zu einer Schule für Gartenbau.

Eck dagegen vertrat die Meinung, es werde viel zu wenig Wert auf Landschaftsgärtnerei gelegt, hierauf müssten wöchentlich zehn bis zwölf Unterrichtsstunden entfallen, ein bloßes Kopieren von Plänen sei ungenügend, die Schüler müssten auch selbständig Pläne entwerfen.

Im weiteren Gespräch wurde seitens des Ministeriums mitgeteilt, dass »[der] Plan [der Gründung einer Akademie] aber vollständig aufgegeben worden sei, nachdem man sich davon überzeugt habe, daß die Zahl der Stellen für akademisch gebildete Gärtner in Sachsen jedenfalls zu gering sei«?

Bouché umriß daraufhin noch einmal das Anliegen der Schule hinsichtlich der Ausbildung von Landschaftsgärtnern und betonte, dass an den Landschaftsgärtner ziemlich hohe Anforderungen gestellt würden, er müsse bewandert sein in Mathematik, Landschaftszeichnen, Plan-Entwerfen und Projektionslehre. Das erfordere eine gründliche Vorbildung, wie sie nur durch ein akademisches Studium erreichbar sei. Derartig vollkommen ausgebildete Gärtner zu erziehen sei bei einem zweijährigen Unterricht ausgeschlossen und läge auch nicht in der Absicht der Schule.

»Wenn die Brauchbarkeit der Schüler in der Praxis als Probe auf die Leistungsfähigkeit der Schule gelten könne, so habe seiner Überzeugung nach die Gartenbauschule diese Probe durchaus befriedigend bestanden. Er habe selbst wiederholt ehemalige Schüler in seinem Bureau sowie mit praktischen Arbeiten beschäftigt und sei mit ihren Leistungen ganz zufrieden gewesen. Die betreffenden Leute hätten sich als durchaus verwendbar und tüchtig erwiesen. Auch andere, die frühere Schüler beschäftigen, hätten deren Brauchbarkeit gerühmt . $^{8}$

Seidel bestätigte das und ergänzte, >das allgemeine Ziel sei nicht der Obergärtner, sondern tüchtige Gehilfen, die sich in den ver- 
schiedensten Verhältnissen des Berufes zurechtfinden auf einer gediegenen Grundlage des Wissens, deren weiterer Ausbau aber Aufgabe des Einzelnen sei. $\iota^{9}$

Grundsätzlich gab es am Ende des Gesprächs allgemeine Zustimmung zur Entwicklung der Schule. Von Bedeutung ist das Gespräch außerdem in zweifacher Hinsicht. Zum einen konnte nicht auf eine baldige Gründung einer Akademie für Gartenkunst gehofft werden, zum anderen wird aber deutlich, dass bereits in den Anfangsjahren ein großes Interesse an der Ausbildung in der Landschaftsgärtnerei bestand.

Im Nachgang zu diesem Gespräch veranlasste das Ministerium am 23. November 1896, dass Delegierte aus der Gärtnerschaft die Schule prüften. Dazu wurden Vertreter aus dem Erzgebirgischen Gartenbauverein, dem Leipziger Gärtnerverein und dem Obst- und Gartenbauverein Zittau benannt. Die Urteile waren überwiegend positiv. Bemerkenswert ist, dass einige Delegierte bis dato nur ungenügende Kenntnis über die Schule hatten, so zum Beispiel der Königliche Garteninspektor Wilhelm Mönkemeyer vom Botanischen Garten in Leipzig, der ausdrücklich einräumte, >dass er die Schule bisher nur ungenügend gekannt habe, jetzt aber von deren Wert vollständig überzeugt sei. ${ }^{10}$ Seitens der Delegierten wurde angeregt, die Situation bei den Lehrmitteln zu verbessern und einen Winterlehrgang für weniger vorgebildete Gärtnergehilfen einzuführen. Insgesamt ging die Schule gefestigt aus dieser Überprüfung hervor.

Diese gefestigte Position kam auch im fünften Bericht zur Schule für die Jahre 1897/98 zum Ausdruck, wo eingeräumt wurde, dass ursprünglich Bedenken aus der Gärtnerschaft gegenüber der Schule bestanden hatten, während es nun Sympathie für die Schule gab. Das galt besonders für die Neuerung, dass jede praktische Arbeit ausgeschlossen war. >Diese Beschränkung hat sich aufs Beste bewährt und der Einfluß der Pomologie ist hier im Gegensatz zu anderen Einrichtungen nicht zu groß. ${ }^{11}$
Die Zustimmung ist auch daraus ersichtlich, dass die Stadt Dresden Ende November 1897 beschloss, zunächst für drei Jahre je 1.000 Mark für die Finanzierung der Schule beizusteuern.

\section{9-1906}

1899 gab es infolge des Todes von Lämmerhirt am 28. November eine wichtige Personaländerung. Fritz Tamms, bis dahin Leiter der Kolbeschen Obstkulturen in Radebeul, wurde an die Schule berufen und der Unterricht lückenlos fortgesetzt.

Schrittweise wurde die Ausbildung in der Landschaftsgärtnerei vertieft, was sicher auch eine Reaktion auf das hohe Interesse der Schüler darstellte.

Zur Landschaftsgärtnerei hieß es im zehnten (Jubiläums-)Bericht 1902/1903:

"In der Landschaftsgärtnerei sah man unter den Gartenplänen recht flott gezeichnete Arbeiten in den verschiedensten Manieren und zumeist mit guter Technik hergestellt. Namentlich bewiesen vielen Fleiß die sogenannten Arbeitspläne für kleinere und größere Anlagen, in welchen die alte Lage des zu behandelnden Grundstücks deutlich erkennbar und darüber aber der Entwurf für die Veränderungen in kräftigem kontrastierenden Kolorit eingezeichnet wird. Diese mühsam herzustellenden Zeichnungen schärfen den Blick des Schülers für die Neuanlagen und erleichtern ihm sein Verständnis für die Ausführung im Freien. Auch die Ausarbeitung von Terrainplänen findet im Anschluß an den Feldmeßunterricht die eingehende Behandlung beim technischen Zeichenunterrichte, in Verbindung damit stehen Berechnungen der Horizontalkurven, Darstellungen von Längs- und Querprofilen und die Bestimmung der zu bewegenden Bodenmassen. Die eigenen Entwürfe der Schüler von Hausgärten nach gegebenen Lagen zeigten oft nennenswerte, nicht zu unterschätzende Fähigkeiten 
auch auf diesem Gebiet. Die gediegene, für die Praxis berechnete Ausbildung unserer Schüler auch in diesen Fächern erklärt die Thatsache, daß unsere Schüler von Landschaftsgärtnern und Gartenkünstlern gern genommen werden und gesuchte Hilfskräfte abgeben. Viele unserer ehemaligen Schüler sind heute als selbständige Landschaftsgärtner, oder bei Verwaltungen öffentlicher Anlagen, in namhaften Bureaux und landschaftsgärtnerischen Geschäften mit bestem Erfolg thätig «. ${ }^{12}$

1903 trat der Verein ehemaliger Dresdner Gartenbauschüler - er war bereits 1898 gegründet worden - an das Kuratorium der Schule heran und beantragte die Einführung einer unter staatlicher Aufsicht stehenden Obergärtnerprüfung. Außerdem wurde für eine Ausweitung des Unterrichtes zur Landschaftsgärtnerei plädiert. Hierzu aus dem elften Bericht der Schule für 1903/1904:

"Das 12. Schuljahr weist wieder einen sichtbaren Fortschritt in der Entwicklung der Schule auf. Neben den vermehrten Exkursionen zur Ergänzung des Unterrichts in den verschiedenen wissenschaftlichen und technischen Fächern hat der Lehrplan für die Landschaftsgärtnerei eine Erweiterung erfahren müssen. Wie im vorigen Jahresberichte bereits bemerkt wurde, waren die Leistungen im technischen Zeichnen für Gewächshausbau und Planzeichnen für Landschaftsgärtnerei schon bisher sehr erfreulich. Der Umstand, daß die ehemaligen Gartenbauschüler unter genauer Begründung der Notwendigkeit die Schulleitung um eine Erweiterung dieses Unterrichtes ersuchten und zugleich baten, die Landschaftsgärtnerei, welche bisher als Nebenfach im Lehrplan bezeichnet war, zu einem Hauptfache $\mathrm{zu}$ erheben, veranlaßte die Schulleitung und das Kuratorium, diesen Antrag auf seine Berechtigung zu prüfen. Dabei hat sich unter anderem herausgestellt, daß ein ansehnlicher Teil früherer Schüler sich in der Landschaftsgärtnerei betätigt und entweder in städtischen Ver- waltungen, Bureaus von Gartenkünstlern oder auch als selbständige Landschaftsgärtner eine beachtenswerte Praxis ausübt. Diese Tatsache veranlaßte das Kuratorium, dem Gesuche der ehemaligen Schüler zu entsprechen und außerdem den Unterricht in der Landschaftsgärtnerei für jede Klasse um 2 Stunden zu vermehren. Der Erfolg hiervon ist ein sichtbarer und schon heute die Zweckmäßigkeit der Maßnahme zu erkennen ${ }^{13}{ }^{13}$

Nach Prüfung des Antrags empfahl der Landeskulturrat dem Ministerium des Innern, eine Obergärtnerprüfung einzuführen.

\section{7-1914}

Eine tiefgreifende Veränderung erfolgte 1907. Am 18. April 1907 wurde dem Ministerium des Innern - scheinbar auch von diesem angeregt - vom Berufsstand eine »Denkschrift über die Umgestaltung der Gartenbauschule« überreicht. Sie beschrieb zwei wesentliche Missstände der Schule, nämlich - ein jährlich ungünstiges finanzielles Ergebnis und außerdem

- trage die Schule in ihrem Zweck den heutigen Bedürfnissen nicht mehr genügend Rechnung.

Zur Verbesserung gab es folgende Vorschläge:

- Einrichtung eines neuen, einjährigen Kurses (ohne Gartenkunst) mit Betonung der praktischen Fähigkeiten;

- Fortführung des bisherigen zweijährigen Kurses;

- Einführung der Obergärtnerprüfung;

- Verlegung der Schule, um den auftretenden, größeren Raumbedarf zu befriedigen. Das Kuratorium und der Verband hatten dazu beschlossen, das Grundstück "Minnahof" in Laubegast, Poststraße 17, anzumieten, wo neben der Schule auch das Internat eingerichtet werden konnte und ein großer Garten zur Verfügung stand (Abb. 2);

- Einrichtung einer Abendschule für Gärtnergehilfen im Winter, wo Buchführung, 
Rechnen, Deutsch, Geometrie und Zeichnen unterrichtet wurden;

- Zur Begründung wurden auch finanzielle Vorteile angeführt, insbesondere mehr Schulgeldeinahmen und weniger Miete. ${ }^{14}$ Die Eröffnung der neuen Schule erfolgte am 12. August 1907 in Laubegast.

Im Herbst 1907 wurde die Obergärtnerprüfung eingeführt. Voraussetzung für diese zweite Prüfung war der Abschluss des zweijährigen Kurses mit der Note 2 und eine weitere, mindestens vierjährige praktische Tätigkeit. Ziel war der Nachweis der Befähigung zur Übernahme einer leitenden Stelle als Obergärtner. Die Prüfung mit einem mündlichen und einem schriftlichen Teil erfolgte in jedem Fall in gärtnerischer Pflanzenzucht und wahlweise in

- Obst- und Gemüsebau,

- Landschaftsgärtnerei,

- Botanischer und Versuchsgärtnerei.

Außerdem ist erwähnenswert, dass im Dezember 1907 die Vereinigung ehemaliger Dresdner Gartenbauschüler eine Denkschrift mit Verbesserungsvorschlägen für den Unterricht einreichte. Die Vorschläge betrafen in besonderer Weise auch die Lehrfächer zur Landschaftsgärtnerei. Vorgeschlagen wurde unter anderem:

- Verknüpfung der Gartenstile mit den Baustilen;

- Ergänzung der Theorie der Landschaftsgärtnerei unter besonderer Betonung der künstlerischen Seite;

- Aufteilung der Gartenkunst in zwei Lehrgänge:

- Allgemeiner Lehrgang mit Betonung der praktischen Seite

- Besonderer Lehrgang mit Betonung der künstlerischen Seite.

Das Anschreiben zur Denkschrift stammte von Louis Kniese, der die Schule von 1895 bis 1897 besuchte und 1922 als Lehrer für Gartenkunst an der neuen Höheren Staatslehranstalt für Gartenbau Dresden-Pillnitz tätig wurde. Es scheint, dass er die Denkschrift in wesentlichen Teilen selbst verfasste.
Von Jahr zu Jahr wuchs das Interesse der Schüler an der gartenkünstlerischen Ausbildung, so dass sich die Schulleitung gehalten sah, im sechzehnten Bericht für 1908/09 auf den begrenzten Arbeitsmarkt hinzuweisen: »Es ist genugsam bekannt, daß aus den höheren Gärtnerlehranstalten Deutschlands eine sehr große Zahl Landschaftsgärtner hervorgeht, so daß in absehbarer Zeit dieser Zweig der Gärtnerei für nicht speziell Begabte oder unvollkommen Vorbereitete überfüllt sein wird. Außer der Königlichen Gärtnerlehranstalt zu Dahlem stellt unsere Lehranstalt vielleicht die höchste Ziffer. Es gereicht uns gewiß zu hoher Freude, daß die aus unserem Institut hervorgehenden Landschaftsgärtner sich so gut bewähren und sich $\mathrm{zu}$ behaupten vermögen. Die bei unseren Obergärtnerprüfungen geleisteten vorzüglichen Arbeiten, die sich auf unserer Lehranstalt obligatorisch in demselben Maße auf Gartenbau wie auf Gartenkunst erstrecken, sind ein weiterer Beweis dafür. Wir werden nach wie vor dieses Lehrfach auf der Höhe halten und uns alles Neue und Gute zunutze machen ${ }^{15}{ }^{15}$

Ungeachtet dessen blieb das breite Interesse der Schüler an der Landschaftsgärtnerei bestehen. Der achtzehnte Bericht für 1910/11 dokumentierte auch eine Vertiefung in der Ausbildung. Der Unterricht in Freihand- und Linearzeichnen wurde aufgegeben, dafür wurden wöchentlich zwei Stunden Perspektivzeichnen und eine Stunde Aquarellmalen eingeführt. Für diesen Unterricht wurde der Kunstmaler und Zeichenlehrer Max Starke eingestellt. Allgemein führte Bertram zum Unterricht in Landschaftsgärtnerei aus:

»Planzeichnen, Gartentechnik, Feldmessen und Gartenkunst gehen auf unserer Lehranstalt Hand in Hand. Sie sind Hauptfächer, und ein größerer Teil der Schüler kommt schon mit der Absicht zu uns, sich als Gartenbautechniker auszubilden. Wir fördern diese Bestrebungen nach Kräften. Die Ansprüche aber, die 
heute an einen tüchtigen Landschaftsgärtner gestellt werden, sind außerordentlich hohe, und wir müssen deshalb die ernste Mahnung laut werden lassen, daß nur diejenigen sich der Gartenkunst ganz widmen, die von Natur aus eine künstlerische Begabung dafür besitzen, damit sie vor argen Enttäuschungen bewahrt bleiben. [...] [Das] Zeichnen beginnt mit den einfachen mechanischen Bleistiftübungen nach >Bertrams Planzeichnen und endigt mit der möglichst größten Vollendung der Pinseltechnik in verschiedenen Farben. Die Übungen gehen mit dem Nachzeichnen von Plänen Hand in Hand. Das Übertragen der Zeichenvorlagen für Wegekurven und Gehölzgruppen wird von vornherein nach Art der Feldmeßarbeiten geübt, zunächst im Quadratnetz, dann in Form der Triangulation. Alsdann folgen Unterweisungen im Abstecken von Blumengruppen, Schmuckplätzen und vollständigen Gartenanlagen. Ist die erforderliche Fertigkeit im Übertragen von Zeichnungen erreicht, so beginnt die Ausarbeitung größerer Pläne mit allen vorkommenden Nebenzeichnungen, z. B. der Darstellung des Geländes mit Höhenlinien, und bei Umänderungen alter Park- und Gartenanlagen die Darstellung der alten Lage mit der neuen Umänderung «. ${ }^{16}$

Für den Unterricht in Gartentechnik wurde angemerkt, dass das Lehrbuch von Bertram zur Gartentechnik genutzt wurde.

Der Unterricht in Gartenkunst behandelte unter anderem deren Geschichte, die Verbindung zur Architektur, Gehölzgruppierung, die Wegeführung und die Aufgaben der Gartenkunst in den Städten und Gemeinden. Weiterhin schrieb Bertram hierzu:

"Der Zeichenunterricht in diesem Fache beginnt mit dem Entwerfen kleinerer Hausgärten auf gegebenen Grundplänen, beschäftigt sich später mit dem Entwerfen größerer Gärten und Parkanlagen und aller dabei vorkommenden Nebenaufgaben und verlangt schließlich von dem Schüler korrekte Kostenanschläge und Bepflanzungspläne für die eigenen Entwürfe «. ${ }^{17}$

$\mathrm{Zu}$ den neuen Fächern Perspektiv- und Aquarellzeichnen äußerte sich Max Starke wie folgt:

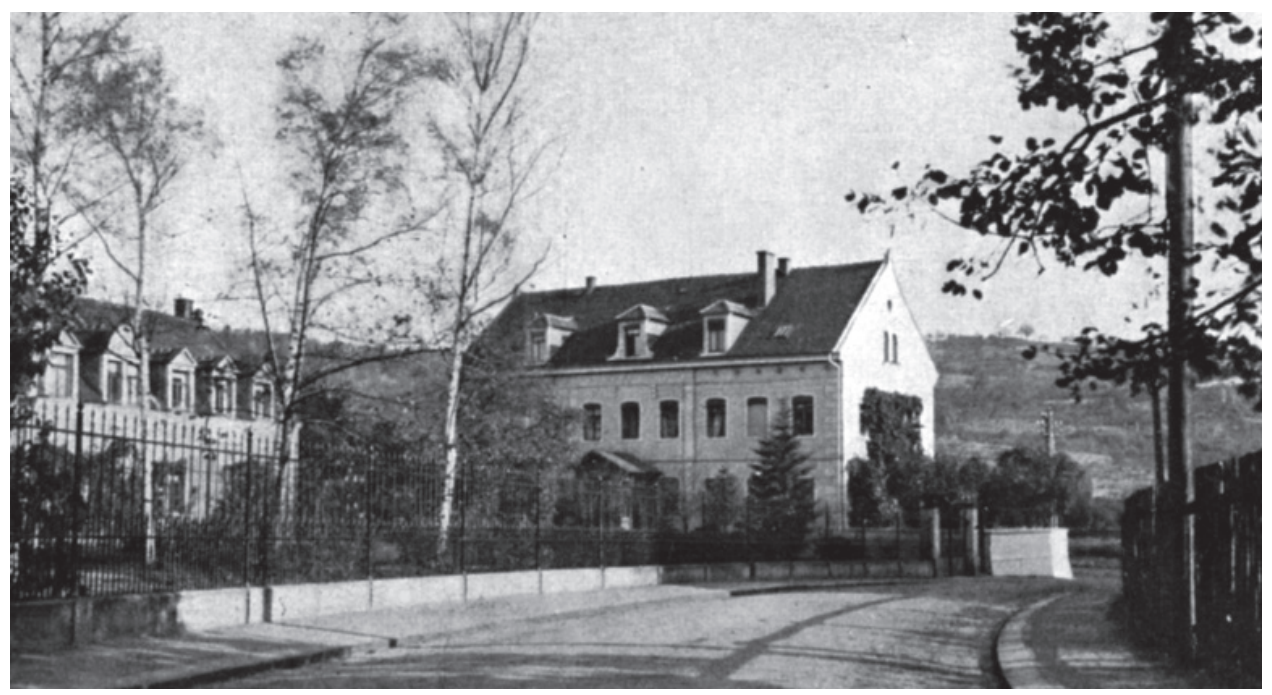

2 | Der »Minnahof« in Laubegast - Sitz der Gartenbauschule ab August 1907. (15. Bericht über die Gartenbauschule des Gartenbau-Verbandes für das Königreich Sachsen). 
$»[\ldots]$ wenn man etwa meint, das Zeichnen perspektivischer Gartenpläne sei nicht gerade als wichtiges Objekt im gärtnerischen Berufe anzusehen, so muß doch diese Ansicht die Tatsache erschüttern, daß sich schon längst der Architekt zum Konkurrenten des Gartenarchitekten gemacht hat, eben durch Kenntnis in diesem Fache. Kein Wunder, dass er damit nur zu oft durch seine perspektiv gezeichneten und gemalten Gartenpläne manchem Gärtner die Aufträge entzogen hat. Dabei muß eben berücksichtigt werden, daß vielen Bestellern durch solche Anschaulichkeit besser gedient werden kann. Infolgedessen sind auch Anfragen von größeren Gärtnereien und Gärtnern mit Kenntnissen in der Herstellung solcher Pläne schon öfters an unsere Anstalt ergangen. Ostern 1910 wurden daher wöchentlich 3 Lehrstunden dafür eingerichtet. Beide Abteilungen beginnen mit dem Zeichnen einfacher, perspektivischer Körper, dann Naturstudien, landschaftliche Silhouetten, einzelne Gartenstudien usw. Endlich sollen Übungen des Handgelenks, Schärfung und Bildung der Augen sowie Beobachtungsgabe nebst Sauberkeit und Ordnungsliebe zur Vertiefung des Geschmacks beitragen . $^{18}$

Exkursionen zu bedeutenden Gartenanlagen wurden fester Bestandteil des Unterrichts zur Landschaftsgärtnerei, zum Beispiel 1911 eine zweitägige Exkursion nach Chemnitz.

Neu war, dass auch Schülerinnen aufgenommen werden konnten, wenn sie die nötige Schulbildung und eine zweijährige Ausbildung in einer guten Gärtnerei nachweisen konnten.

Die Schuljahre 1912/13 und 1913/14 stellten sicher den Höhepunkt in der Geschichte der Laubegaster Gartenbauschule bezüglich Schülerzahl und Qualität der Ausbildung dar. Im Frühjahr 1913 gab es mehr Bewerber als die Schule aufnehmen konnte. Insgesamt waren zusammen mit dem Winterkurs für Gehilfen 50 Schüler an der Schule angemel- det. Der Unterricht wurde von 15 Lehrern erteilt. Zu Planzeichnen, Gartentechnik, Feldmessen und Gartenbaukunst erläuterte der neue Direktor Fritz Tamms im Bericht für 1912/13:

»[...] Der Unterricht in Gartentechnik bringt Vorträge und praktische Unterweisungen für alle bei Ausführung und Unterhaltung der Anlagen erforderlichen Arbeiten, wie Erd- und Wegearbeiten, Wasseranlage, Wasserleitung, Entwässerungen, Schleusenbau und verschiedene andere und wird unterstützt durch das Lehrbuch >Bertrams Gartentechnikı, ein reich illustriertes Werk mit den besten Zeichnungen und Darstellungen des gartenbaulichen Betriebes. [...] Großes Interesse zeigen die Schüler für den Unterricht in der Gartenkunst selbst. Vorträge über Abschnitte aus der Geschichte der Gartenkunst, ihre Entstehung und allmähliche Entwicklung in Verbindung mit der Architektur leiten dieses Lehrfach ein. Ihnen folgen Sondervorträge über Bodenbewegungen vom künstlerischen Standpunkte aus, über Gehölzgruppierung und Wegeführung und schließlich über die Aufgaben der Gartenkunst in Städten und Gemeinden an Hand reichen Demonstrationsmaterials $\ll .{ }^{19}$

Betrachtet man die Entwicklung der gartenkünstlerischen Ausbildung an der Gartenbauschule des Gartenbauverbandes von 1892 bis 1914, so bleibt die Feststellung, dass sie sich - zum Teil gegen die ursprünglichen Intentionen - von einer Ergänzung zum Hauptunterricht zu einem ausgesprochenen Schwerpunkt gewandelt hat. Der Unterricht nahm an Breite und Tiefe deutlich zu. Dadurch ergab sich auch ein anderes Profil der Absolventen. Ursprünglich mit dem Ziel ausgebildet, als Hilfskräfte für Landschaftsarchitekten zu arbeiten, waren sie spätestens ab 1910 so gut ausgebildet, dass viele als selbständige Landschaftsgärtner oder in leitender Stellung bei Gartenverwaltungen oder größeren Landschaftsarchitekturbüros tätig waren. 


\section{4-1918}

Am 9. Juni 1914 starb Max Bertram. Als Vorsitzender des Kuratoriums schrieb Bouché am 23. Juni 1914 an das Ministerium des Innern und teilte mit, dass dadurch der Unterricht in Gartenkunst, Gartentechnik und Feldmessen frei sei. Er schlug für die Nachbesetzung den Garteningenieur und geprüften Obergärtner Louis Kniese vor. Kniese hatte nach seiner Ausbildung an der Gartenbauschule bei Bertram in Dresden, bei Hampel in Berlin und bei Bouché im Großen Garten gearbeitet. Bouché betonte, dass dieser Vorschlag neben der fachlichen Qualifikation auch darauf beruhte, dass Kniese auf Privatarbeiten verzichtete, was die anderen Bewerber nicht zusagen wollten. Die Anstellung war ab 15. August 1914 geplant. Hierzu kam es jedoch nicht, da Kniese zum Militär einberufen wurde.

Durch den Krieg gingen die Schüler- und die Lehrerzahlen stark zurück, der Schulbetrieb konnte aber aufrechterhalten werden. Während der Kriegsjahre hatte die Schule durchschnittlich 10-15 Schüler.

Die Personalliste vom 18. Oktober 1916 wies fünf Lehrer aus, davon hatten vier das Dienstalter für den Militärdienst überschritten. Lehrer für Gartenkunst, Feldmessen und Planzeichnen war Georg Arthur Gruhle, der das Dienstalter noch nicht überschritten hatte. Ursprünglich nicht dienstverwendungsfähig, wurde er 1915 als garnisonsver- wendungsfähig eingestuft, so dass ihm die Einberufung drohte. Tamms notiert in der Personalliste, die an das Ministerium zu melden war und offensichtlich auch dazu diente, diejenigen $\mathrm{zu}$ ermitteln, die nicht unbedingt für die Aufrechterhaltung des Betriebes nötig waren und deshalb zum Wehrdienst einberufen werden konnten:

»Herr Gruhle ist Lehrer für unser Hauptfach `Gartenkunst`. Seit 1915, wo er als garnisonsverwendungsfähig bezeichnet wurde, war die Schulleitung nach Kräften bemüht, einen Ersatzmann zu finden und heute ist es geradezu unmöglich, eine auch nur leidlich geeignete Lehrkraft zu bekommen. Fiele dieses Lehrfach aber aus, so wäre die Weiterführung der Schule in Frage gestellt $\ll^{20}$

Diese Darstellung hatte tatsächlich Erfolg. Es gab ein entsprechendes Schreiben des Ministeriums des Innern an das stellvertretende Generalkommando des XII. Armeekorps vom November 1916 und dann Anfang Dezember 1916 die positive Antwort an die Schule.

Im Juni 1917 folgte ein weiteres Gesuch, den Kunstmaler Starke wieder vom Militärdienst freizustellen, ebenfalls mit Erfolg, er wurde am 18. August 1917 für den Zivildienst entlassen.

Alles in allem überlebte die Schule die Kriegsjahre und ging ab 1922 in der neu gegründeten »Höheren Staatslehranstalt für Gartenbau in Pillnitz« auf.
1 Bertram, Max: Erster Bericht über die Gartenbauschule des Gartenbau-Verbandes für das Königreich Sachsen, E. G. zu Dresden für die Jahre 1892/93 und 1893/94, Dresden 1894.

2 Ebd., S. 6.

3 SächsHStAD, 10736 Ministerium des Innern, Nr. 15805, Bl. 77.

4 Ebd.

5 Ebd., Bl. 87.

6 SächsHStAD, 10736 Ministerium des Innern, Nr. 15806, Bl. 15.

7 Ebd., Bl. 16

8 Ebd., Bl. 17

9 Ebd.

10 Ebd., Bl. 27.
11 Ebd., Bl. 77, gehefteter Jahresbericht, S. 3 f.

12 Ebd., Bl. 201, gehefteter Jahresbericht, S. 14f.

13 SächsHStAD, 10736 Ministerium des Innern, Nr. 15807, Bl. 48, gehefteter Jahresbericht S. 3.

14 Ebd., Bl. 101-106.

15 SächsHStAD, 10736 Ministerium des Innern, Nr. 15808, Bl. 53, gehefteter Jahresbericht, S. 9.

16 Ebd., Bl. 122, gehefteter Jahresbericht, S. 17f.

17 Ebd., Bl. 122, gehefteter Jahresbericht, S. 18.

18 Ebd., Bl. 122, gehefteter Jahresbericht, S. 24.

19 SächsHStAD, 10736 Ministerium des Innern, Nr. 15809, Bl. 45 , gehefteter Jahresbericht, S. $20 \mathrm{f}$.

20 Ebd., Bl. 138 